\title{
Valor pronóstico de la asociación entre la expresión de maspin y la ocurrencia de apoptosis en tumores mamarios caninos
}

\author{
Pantiú, A.J.'; Insfrán, R.M.'; Merlo, W.A..; Ortega, H.H. ${ }^{2}$; Rosciani, A.S. ${ }^{1}$
}

${ }^{1}$ Servicio de Diagnóstico Histopatológico y Citológico, Facultad de Ciencias Veterinarias, Universidad Nacional del Nordeste, Sargento Cabral 2139, Corrientes (3400), Argentina, Tel. 00543783425753 Int. 173. ${ }^{2}$ Cátedra de Biología Celular, Facultad de Ciencias Veterinarias, Universidad Nacional Litoral. E-mail: arosciani@vet.unne.edu.ar

\begin{abstract}
Resumen
Pantiú, A.J.; Insfrán, R.M.; Merlo, W.A.; Ortega, H.H.; Rosciani, A.S.: Valor pronóstico de la asociación entre la expresión de maspin y la ocurrencia de apoptosis en tumores mamarios caninos. Rev. vet. 22: 2, 95-99, 2011. La proteína maspin (inhibidor mamario de proteasa de serina) en la especie humana es considerada como supresor tumoral, atribuyéndosele distintos mecanismos de acción, entre ellos la inducción a la apoptosis, por lo que en este trabajo se investigó la ocurrencia de asociación entre la presencia de proteína maspin y apoptosis en carcinomas mamarios de la perra, así como su relación con la evolución de las pacientes. Se trabajó con neoplasias mamarias espontáneas de 38 pacientes caninas cuya evolución fue controlada hasta dieciocho meses postcirugía. Se realizó el diagnóstico, la graduación histológica de malignidad y el recuento de las figuras apoptóticas (por medio de una grilla cuadriculada de $1 \mathrm{~cm}$ de lado, dividida en 100 áreas de $1 \mathrm{~mm}^{2}$ cada una). Para la inmunomarcación de maspin se empleó el método de biotina-streptavidina-peroxidasa y un anticuerpo monoclonal anti-maspin. Por regresión logística se halló correlación entre la expresión de maspin en el citoplasma y la ocurrencia de apoptosis (p: 0,045; r: 0,31). Al realizar el análisis de la varianza de las medias de apoptosis entre las pacientes vivas $(7,47)$ y muertas $(3,69)$ con citoplasmas negativos a la marcación de maspin, se encontraron diferencias significativas $(p=0,033)$. De acuerdo con lo observado, podría esperarse una mayor sobrevida de las pacientes ante la ausencia de maspin en el citoplasma de las células y valores elevados de apoptosis.
\end{abstract}

Palabras clave: canino, neoplasias mamarias, maspin, apoptosis, pronóstico.

\begin{abstract}
Pantiú, A.J.; Insfrán, R.M.; Merlo, W.A.; Ortega, H.H.; Rosciani, A.S.: Prognostic value of the association between maspin expression and apoptosis in canine mammary tumors. Rev. vet. 22: 2, 95-99, 2011. Maspin protein (mammary serine protease inhibitor) in humans is considered a tumoral suppressor with different mechanisms of action such as apoptosis. For this work we studied the association of occurrence between the presence of maspin protein and apoptosis in canine mammary carcinomas, as well as its relationship with the evolution of patients. We worked with spontaneous mammary carcinomas from 38 bitches that were followed up to 18 months post surgery. Diagnosis and malignancy histological graduation were carried out. Apoptotic bodies were counted by means of a squared grid of $1 \mathrm{~cm}$ side, divided into 100 areas of $1 \mathrm{~mm}^{2}$ each. Maspin immunohistochemistry was performed with a monoclonal antibody using streptavidin-biotin immunoperoxidase system for immunolabeling. Relationship between cytoplasmic maspin expression and apoptosis occurrence was found through logistic regression (p: 0.045; r: 0.31). Patients with maspin negative cytoplasmic stain showed significance (p: 0.033 ) in apoptosis mean analysis of variance between alive (7.47) and dead (3.69) patients. According to these results, we can expect longer survival of patients with the absence of maspin in cells' cytoplasm and high values of apoptosis.
\end{abstract}

Key words: bitch, mammary neoplasms, maspin, apoptosis, prognostic. 


\section{INTRODUCCIÓN}

Las neoplasias mamarias son los tumores más frecuentes en las hembras caninas. Se manifiestan como nódulos únicos o múltiples, que pueden pertenecer al mismo o a diferentes tipos histológicos y afectar una o diversas glándulas. Son llamativos por su estructura y comportamiento biológico variables. Se considera una relación entre neoplasias malignas y benignas de 3:7 o 4:6, con un rango de supervivencia luego de las cirugías de tumores malignos de entre 4 y 17 meses ${ }^{11}$.

El desarrollo del cáncer es un proceso en el que no sólo interviene la desrregulación de factores proliferativos y la activación de oncogenes, sino también la desrregulación de factores de inhibición del crecimiento celular y la pérdida de función genética supresora. En oncología moderna reviste gran interés la valoración de la cinética celular. El estudio de parámetros que reflejan las fases del ciclo en que se encuentran las células neoplásicas, ha demostrado gran utilidad en la evaluación del comportamiento biológico de los tumores ${ }^{13}$.

La apoptosis es un modo de muerte celular programada, responsable de la eliminación de células en los tejidos normales, pero también ocurre en contextos patológicos específicos ${ }^{7}$. En el adulto, la muerte celular programada es la responsable de que se mantenga el equilibrio con la proliferación celular y de conservar constante el número de células en aquellos tejidos sometidos a un continuo recambio celular. Además constituye un mecanismo de defensa mediante el cual las células alteradas, potencialmente peligrosas, son eliminadas ${ }^{1}$. En las neoplasias mamarias malignas de la especie humana, se observa incremento de apoptosis ${ }^{8}$ que se relaciona con un mal pronóstico ${ }^{20} \mathrm{y}$ en los tumores mamarios caninos, se informan resultados similares ${ }^{19}$. En la región, se encuentran trabajos descriptivos que comunican una tendencia al aumento de imágenes de apoptosis en los carcinomas simples ${ }^{15}$.

El inhibidor mamario de proteasa de serina (maspin) es una proteína de $42 \mathrm{kDa}$ que pertenece a la superfamilia de inhibidores de proteasa de serina (serpin). Se la encuentra en la glándula mamaria normal de la mujer (también en otros epitelios), es producida principalmente por las células mioepiteliales y su presencia disminuye hasta desaparecer en el cáncer. Se informa que el gen p53 activa la expresión de esta proteína inhibidora ${ }^{22}$. Otros autores establecen que maspin es un inhibidor efectivo de la angiogénesis, capaz de reducir la densidad de la microvasculatura y de esta manera suprimir el crecimiento tumoral in vivo e in vitro ${ }^{21}$. Se postula que su expresión resulta en una disminución de la motilidad celular e invasión, concomitante con un incremento del índice de apoptosis ${ }^{2}$, aunque otros trabajos refieren que esta proteína actuaría principalmente inhibiendo la invasión y no induciendo a la apoptosis ${ }^{16}$. Se asevera que pacientes con falta de expresión de maspin tienen mayor riesgo de desarrollar metástasis, asignándole valor pronóstico a su determinación ${ }^{9}$. En este sentido, algunos autores consideran relevante establecer su localización subcelular (en núcleo o citoplasma) ${ }^{17}$.

Otros trabajos contradicen lo expuesto y describen una sobreexpresión de maspin en las células carcinomatosas, relacionada con un pronóstico desfavorable en tumores de glándula mamaria, así como en carcinomas de páncreas y ovario $9,17,18$.

Se ha informado la presencia de maspin en tejido mamario canino, tanto normal como neoplásico, especificando localización nuclear y citoplasmática de esta proteína en las células mioepiteliales y sólo citoplasmática y en menor grado, en las células carcinomatosas ${ }^{3}$. Se ha destacado una asociación entre la escasa expresión de maspin y la ocurrencia de metástasis, así como la relación entre una moderada y fuerte marcación positiva de los núcleos y un buen pronóstico para las pacientes caninas ${ }^{23}$.

Luego del diagnóstico de una neoplasia, resulta de suma importancia establecer un pronóstico acerca de la posible evolución del paciente. Se han realizado diversos trabajos con el objeto de determinar factores de valor pronóstico para las neoplasias mamarias caninas. Se consideran indicadores de comportamiento biológico tumoral al: tipo histológico, graduación tumoral histopatológica ${ }^{12}$, cinética tumoral que comprende indice de proliferación y apoptosis ${ }^{19}$, estadificación clínica, tamaño y angiogénesis tumoral ${ }^{5,14}$.

Se han establecido diferentes pruebas estadísticas multivariadas para la identificación de factores independientes, siendo el tipo histológico del blastoma, uno de los más destacados. Estas determinaciones confirmaron una relación directa entre el alto grado de anaplasia y un pronóstico desfavorable. De todas maneras, a pesar de estos indicadores, el comportamiento biológico de los tumores mamarios caninos es aún difícil de predecir. Por ello es imprescindible la identificación de otras herramientas que permitan reconocer pacientes con alto riesgo de recurrencia y muerte ${ }^{9}$. Se propone que esta dificultad para diseñar un sistema de clasificación que asegure reproducibilidad y categorización pronóstica se debe a la heterogeneidad histomorfológica observada en las neoplasias mamarias caninas ${ }^{4}$.

En esta oportunidad se realizó un estudio retrospectivo sobre pacientes con seguimiento de su evolución, la cual se relacionó con la expresión de proteína maspin, la ocurrencia de apoptosis y el grado histopatológico de los tumores, buscando establecer el valor pronóstico de tales determinaciones. Como objetivo general se consideró determinar la existencia de parámetros que permitan predecir la evolución de las pacientes caninas con neoplasias mamarias.

\section{MATERIAL Y MÉTODOS}

Se trabajó con neoplasias mamarias espontáneas de 38 pacientes caninas (de diversas razas y edad promedio de 9 años) que fueron atendidas en el Hospital de Clínicas de la Facultad de Ciencias Veterinarias de 
la UNNE (Corrientes, Argentina) y cuya evolución fue seguida durante 18 meses posteriores a la cirugía.

Sobre cortes obtenidos por medio de la técnica histológica clásica y coloreados con hematoxilina y eosina, se realizó la graduación histológica de malignidad de los tumores de acuerdo con lo establecido por Misdorp et al. (2002) ${ }^{12}$. La misma tiene en cuenta la arquitectura, diferenciación celular y número de figuras mitóticas, determinando la existencia de tres grados posibles.

El recuento de figuras apoptóticas se efectuó a 400 aumentos, por medio de una grilla cuadriculada de 1 $\mathrm{cm}$ de lado, dividida en 100 áreas de $1 \mathrm{~mm}^{2}$ cada una, que se colocó en el ocular (1421A de American Optical Corporation). Se contabilizaron las áreas en las que se observaron imágenes de apoptosis.

Para la inmunomarcación de la proteína maspin se realizaron cortes histológicos con micrótomo rotativo de tipo Minot a $3 \mu \mathrm{m}$ de espesor, montados sobre portaobjetos pretratados con una solución de Poly-L-Lysine (diluida 1:10 en agua deionizada). Se requirió de una recuperación antigénica adicional por medio de la incubación en buffer EDTA a $90^{\circ} \mathrm{C}$ durante $30 \mathrm{~min}$. Luego se inactivó la peroxidasa endógena y se bloquearon uniones inespecíficas con suero normal de cabra (ya que el anticuerpo secundario empleado fue desarrollado en esta especie). Finalmente, los distintos cortes se incubaron con el anticuerpo primario. Se utilizó el anticuerpo monoclonal de ratón anti-maspin (G167-70, BD-Pharmingen, USA) incubando toda la noche a temperatura ambiente. Para el revelado se empleó el método de biotina-streptavidina-peroxidasa (Supersensitive Immunodetection System, LP000-UL, Biogenex, CA, USA). Se siguieron los lineamientos generales de los laboratorios proveedores de los distintos anticuerpos.

Para la interpretación de la inmunomarcación se empleó el método de Sood et al. (2002) ${ }^{17}$ el cual tiene en cuenta el porcentaje de células marcadas y la intensidad, cuyos puntos se suman y dan un índice final (OMS: overall maspin score) que considera cuatro rangos: negativo (0), débil (1), moderado (2) y fuerte (3). Se discriminó entre marcación de núcleos y citoplasmas. Para el análisis estadístico, los grupos de OMS 0 y 1 fueron considerados negativos a maspin, mientras que los OMS 2 y 3 se interpretaron como positivos. Se empleó el programa Infostat/Profesional, versión 1.1.

\section{RESULTADOS}

Teniendo en cuenta la evolución de las pacientes estudiadas se formaron dos grupos según la sobrevida a los dieciocho meses: pacientes vivas ( $n 16)$ y pacientes muertas ( $n$ 22). La graduación histológica de malignidad (GH) evidenció 29 neoplasias del grado histológico uno (GHI) y 9 correspondientes al grado histológico dos (GHII), no habiéndose presentado casos del grado
Tabla 1. Presencia de maspin en núcleo y citoplasma en relación con el grado histológico de malignidad y la evolución.

\begin{tabular}{lcccc}
\hline & \multicolumn{2}{c}{ maspin en el núcleo } & \multicolumn{2}{c}{ maspin en el citoplasma } \\
\cline { 2 - 5 } & negativo & positivo & negativo & positivo \\
\hline GHI & 19 & 10 & 14 & 15 \\
GHII & 8 & 1 & 7 & 2 \\
vivas & 13 & 3 & 6 & 10 \\
muertas & 14 & 8 & 15 & 7 \\
\hline
\end{tabular}

GHI: grado histológico de malignidad I; GHII: grado histológico de malignidad II.
Tabla 2. Valores promedio y medidas de resumen de apoptosis en relación con la marcación de maspin, el grado histológico de malignidad y la evolución de las pacientes.

\begin{tabular}{lccccc}
\hline & & \multicolumn{4}{c}{ apoptosis } \\
\cline { 3 - 6 } & alternativa & $\mathrm{n}$ & promedio & $\mathrm{DE}$ & $\mathrm{CV}$ \\
\hline \multirow{2}{*}{ maspin en núcleo } & - & 27 & 4,2 & 3,27 & 72,48 \\
& + & 11 & 5 & 3,44 & 67,35 \\
\hline maspin en & - & 21 & 4,77 & 3,76 & 78,83 \\
citoplasma & + & 17 & 4,58 & 2,70 & 58,87 \\
\hline grado histológico & GHI & 29 & 4,88 & 3,52 & 72,19 \\
de malignidad & GHII & 9 & 4,07 & 2,45 & 60,12 \\
\hline \multirow{2}{*}{ evolución } & Vivas & 16 & 5,41 & 3,88 & 71,75 \\
& Muertas & 22 & 4,16 & 2,75 & 66,08 \\
\hline
\end{tabular}

DE: desvío estándar; CV: coef. de variación; -: marcación negativa; +: marcación positiva; GHI: grado histológico de malignidad I; GHII: grado histológico de malignidad II.

tres. Al comparar la sobrevida de las pacientes con el $\mathrm{GH}$, no se hallaron diferencias significativas y su distribución se muestra en la Figura 1.

En la Tabla 1 se observa la frecuencia absoluta de la inmunomarcación de maspin, tanto en núcleo como citoplasma, en relación con el GH y la evolución. Del análisis de los datos obtenidos, no surgió asociación por $\mathrm{Chi}^{2}$ entre la marcación de maspin y el GH o la evolución de las pacientes $(\mathrm{p}>0,05)$.

En la Tabla 2 se presentan los valores promedio de figuras de apoptosis de acuerdo con maspin en sus distintas localizaciones, con el GH y con la evolución. Por regresión logística se hallaron valores significativos al

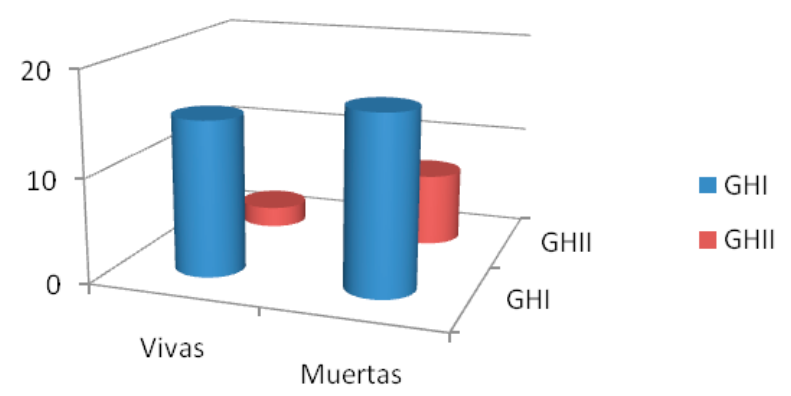

Figura 1. Graduación histológica de malignidad en relación con la evolución de las pacientes. GHI: grado histológico de malignidad I; GHII: grado histológico de malignidad II. 
Tabla 3. Promedios de apoptosis observados en los tumores positivos y negativos a maspin en el citoplasma de acuerdo con la evolución de las pacientes.

\begin{tabular}{lcc}
\hline maspin citoplasma & evolución & promedio apoptosis \\
\hline \multirow{2}{*}{ positivo } & vivas & 4,18 \\
& muertas & 5,16 \\
\hline \multirow{2}{*}{ negativo } & vivas & $7,47^{*}$ \\
& muertas & $3,69^{*}$ \\
\hline
\end{tabular}

* Valores significativos.

relacionar apoptosis con maspin en el citoplasma ( $\mathrm{p}$ : $0,045 ; \mathrm{r:} 0,31)$.

La Tabla 3 muestra los promedios de apoptosis y la marcación de maspin citoplasmático teniendo en cuenta la evolución de las pacientes. Se encontraron diferencias significativas (p: 0,033) entre los promedios de apoptosis observados en pacientes vivas $(7,47)$ y muertas $(3,69)$ con citoplasmas negativos a la marcación de maspin.

\section{DISCUSIÓN}

Diversos trabajos destacan la importancia de la determinación de maspin, aunque existen contradicciones en cuanto a su valoración. En los carcinomas mamarios de la mujer su presencia se asocia con carcinomas de bajo grado y escasa neovascularización ${ }^{22}$ y su ausencia en células carcinomatosas ${ }^{6}$. Otras publicaciones, en cambio, describen una sobreexpresión de maspin en las células carcinomatosas, relacionada con mal pronóstico en tumores de glándula mamaria, así como en carcinomas de páncreas y ovarios de la especie humana ${ }^{18,17}$. En la especie canina se postula que la marcación débil de maspin se asocia con la muerte de las pacientes ${ }^{23}$. En este trabajo, se observó la presencia de maspin en todos los casos analizados.

En cuanto a la localización subcelular de este marcador, no se encontraron diferencias al comparar grado histológico de malignidad ni evolución de las pacientes. No obstante, se halló una relación entre maspin en el citoplasma y los valores promedio de apoptosis. Teniendo en cuenta los mismos en los casos sin maspin en el citoplasma, se observaron diferencias significativas entre las medias de las pacientes que sobrevivieron a los 18 meses y las que murieron, presentándose el promedio más elevado de apoptosis en las pacientes vivas. En este sentido, cobra importancia la determinación subcelular de la marcación de maspin en coincidencia con lo propuesto por otros autores ${ }^{17,23}$ quienes atribuyen mejor pronóstico a la positividad de los núcleos. Sin embargo, nuestros hallazgos son significativos sólo cuando se vincula la ausencia de esta proteína en el citoplasma con los valores promedio elevados de apoptosis.

Por ello, considerando los resultados aquí obtenidos, podría esperarse una mayor sobrevida de las pacientes ante la ausencia de maspin en el citoplasma de las células neoplásicas y valores elevados de apoptosis.

\section{REFERENCIAS}

1. Cooper G. 1997. Cancer. In: The cell. A molecular approach (Cooper G. ed), ASM Press, Sunderland, p. 599632.

2. Ellis ZK, Hendrix MJ. 2003. Nitric oxide regulation of maspin expression in normal mammary epithelial and breast cancer cells. Am J Pathol 162: 1411-1417.

3. Espinosa A, Millán MY, Ramírez GA, Ordás J, Reymundo C, Martin J. 2005. Expression of maspin in mammary gland tumors of the dog. Vet Pathol 42: 250-257.

4. Gilbertson SR, Kurzma ID, Zachrau RE, Hurvitz AI, Black MM. 1983. Canine mammary epithelial neoplasms: biologic implications of morphologic characteristics assessed in 232 dogs. Vet Pathol 20: 127-142.

5. Graham JC, Myers RK. 1999. The prognostic significance of angiogenesis in canine mammary tumors. $J$ Vet Intern Med 13: 416- 418.

6. Hojo T, Akiyama Y, Nagasaki K, Maruyama K, Kikuchi K, Ikeda T, Kitajima M, Yamaguchi K. 2001. Association of maspin expression with the malignancy grade and tumor vascularization in breast cancer tissues. Cancer Letters 171: 103-110.

7. Kerr JF, Winterford CM, Harmon BV. 1994. Apoptosis. Cancer 73: 2013-2026.

8. Lipponen P, Aaltomaa S, Kosma VM, Syrjanem K. 1994. Apoptosis in breast cancer as related to histopathological characteristics and prognosis. Eur J Cancer 14: 2068-2073.

9. Maass N, Takashi H, Rösel F, Ikeda T, Jonat W, Nagasaki K. 2001. Down regulation of the tumor suppressor gene maspin in breast carcinoma is associated with a higher risk of distant metastasis. Clin Biochem 34: 303-307.

10. Madewell BR. 2001. Cellular proliferation in tumors: a review of methods, interpretation, and clinical applications. $J$ Vet Intern Med 15: 334-340.

11. Misdorp W, Else RW, Hellmen E, Lipscomb TP. 1999. Histological classification of mammary tumors of the dog and the cat, 2nd series, Vol. VII, Published by Armed Forces Inst Pathol \& WHO, Washington DC, p. 58.

12. Misdorp W. 2002. Tumors of the mammary gland. In: Tumors in domestic animals (Meuten DJ, ed), $4^{\circ}$ ed., Blackwell, Iowa, p. 575-606.

13. O'Reilly SM, Richards MA. 1992. Clinical aspects of assessing cell proliferation. In: Assessment of cell proliferation in clinical practice (Hall PA, Levison DA, Wright NA, ed.), Springer, London, p. 63-79.

14. Restucci B, De Vico G, Maiolino P. 2000. Evaluation of angiogenesis in canine mammary tumors by quantitative platelet endothelial cell adhesion molecule immunohistochemistry. Vet Pathol 37: 297-301.

15. Rosciani AS, Merlo WA, Ortega HH, Insfrán RM, Sánchez Negrette M. 2008. Determinación del antígeno nuclear de proliferación celular (PCNA) y de apoptosis en tumores malignos de la glándula mamaria canina. Ann Reun Comunic Cient \& Tecn UNNE (Corrientes, Argentina), p. V-30.

16. Shao ZM, Nguyen M, Alpaugh ML, O'Conell JT, Barsky SH. 1998. The human myoepithelial cell exerts antip- 
roliferative effects on breast carcinoma cells characterized by 21 WAF1/CIP1 induction, G2/M arrest and apoptosis. Exp Cell Res 241: 394-403.

17. Sood A, Fletcher MS, Gruman LM, Coffin JE, Jabbari S, Khalkhali Z, Arbour N, Seftor EA, Hendrix MJ. 2002. The paradoxical expression of maspin in ovarian carcinoma. Clin Cancer Res 8: 2924-2932.

18. Umekita Y, Ohi Y, Sagara Y, Yoshida H. 2002. Expression of maspin predicts poor prognosis in breast cancer patients. Int J Cancer 100: 452-455.

19. Yang Y, Liu CH, Chang CJ, Lee CC, Chang KJ, Lin CT. 2006. Proliferative activity apoptosis and expression of oestrogen receptor and Bcl-2 oncoprotein in canine. mammary gland tumours. J Comp Pathol 134: 70-79.

20. Zhang GJ, Kimijima I, Abe R, Watanabe T, Kanno M, Hara K, Tsuchiya A. 1998. Apoptotic index correlates to $\mathrm{Bcl}-2$ and 53 protein expression, histological grade and prognosis in invasive breast cancers. Anticanc Res 18: 1989-1998.

21. Zhang M, Volpert O, Shi YJ, Bouck N. 2000. Maspin is an angiogenesis inhibitor. Nat Med 6: 196-199.

22. Zou Z, Gao C, Nagaich AK, Connell T, Saito S, Moul JW, Seth P, Appella E, Srivastava S. 2000. p53 regulates the expression of the tumor suppressor gene maspin. J Biol Chem 275: 6051-6054.

23. Zuccari DA, Castro R, Gavioli AF, Mancini UM, Tajara EH, Frade CS, Pivaro LR, Carmona J, Terzian AC, Ruiz CM, Goloni EM, Pavarino E. 2009. The maspin expression in canine mammary tumors: an immunohistochemical and molecular study. Pesq Vet Bras 29: 167-173.

\section{Asociación Cooperadora de la Facultad de Ciencias Veterinarias Universidad Nacional del Nordeste}

Personería Jurídica No 647/92 y 912/00

Sargento Cabral 2139

3400 Corrientes

Argentina

La Asociación Cooperadora de la Facultad de Ciencias Veterinarias de la UNNE fue constituida el 10 de diciembre del año 1991 como entidad de bien público, con el objeto de promover y coadyuvar las actividades científicas, educativas y culturales relacionadas con las ciencias veterinarias. En tal sentido, implementa acciones para colaborar con la enseñanza, extensión, actualización y difusión científica que realiza dicha casa de estudios.

\section{Beneficios que brinda a sus asociados:}

- Fotocopias con descuentos especiales del 20\% en la Fotocopiadora Copias.com que funciona dentro del predio de la Facultad de Ciencias Veterinarias.

- Descuentos del 10\% para la adquisición de libros de la Editorial Inter-Médica.

- Descuentos especiales en otros rubros.

Sea solidario, asóciese en beneficio de toda la comunidad universitaria

Tel. 03783-425753 interno 186 • Página Web www.vet.unne.edu.ar•E-mail: cooperadora@vet.unne.edu.ar 